\title{
Implementation of Aerial Panoramic Photography for Environmental Studies Through VR Experiences
}

\author{
Wei Zhong Feng ${ }^{1}$ \\ ${ }^{1}$ Department of Visual Communication, Chaoyang University of Technology, Taichung, 436, Taiwan, ROC. \\ Correspondence: Wei Zhong Feng, Department of Visual Communication, Chaoyang University of Technology, \\ Taichung, 436, Taiwan, ROC. E-mail: feng.jimmy@ msa.hinet.net
}

Received: August 24, 2021

Accepted: September 16, 2021

Online Published: September 27, 2021

doi:10.20849/jess.v4i1.926

URL: https://doi.org/10.20849/jess.v4i1.926

\begin{abstract}
Technology allows us to experience situations from a different perspective. This project investigated many parts and techniques for better VR experiences. Aerial photography: Aerial photography provides a broad view of the changes in a landscape. However, aerial photography cannot offer a microscopic perspective of the changes with novel panoramas and landscape photos. A parallax compensation algorithm for stitching videos enables the user to reduce the time required to fix stitching issues in post-production. The experiment results indicated that the optimum camera height was $500 \mathrm{~m}$, slightly higher than the clouds. An appropriate number of spatial samples were selected during photography to obtain high-quality images. The researchers avoided restricted flight areas and designed the image acquisition method according to the selected flight path, which were then combined in the post-processing step. Those 360-degree-aerial-images trigger young people's environmental awareness, which enhances the creator's sense of environmental protection.
\end{abstract}

Keywords: aerial panoramic, VR photography, multi-projector, science education, image processing

\section{Introduction}

With the development of science and technology, VR technology in various industries is growing. Much attention is paid to the research of VR technology in surrounding guidance to support users with valuable information about the local area.

\subsection{Explore Importance of the $V R$}

In Alqahtani and Manolya (2017) and Zhu and Pang (2018), researchers demonstrated an AR app, "IMAP-CampUS" that provides information about the buildings nearby to assist students at Macquarie University in locating places of interest with overlay information. Andri (2019) designed a virtual tour of Management and Science University helps visitors acquired school information by virtual tour. This project also presents the prominent use cases covered by the application and describes the main feature components. Furthermore, the effectiveness and user experiences in 3D Augmented Reality were evaluated to improved user satisfaction.

Panoramic aerial also applied in environmental art creation. Photographs were taken by a drone using a shockproof aerial camera. The photographed landscapes were spherically transformed into a small planet with a sharp contrast between the green and industrial zones (Feng and Chao 2019). During the post-processing, sound fields of breathing were mixed with video, which hinted visitors the direction to explore 3D space with immersive emotion.

The researcher investigated VR application for architectural heritage preservation using Unreal Engine 4. Panorama photographs ensure the seamless integration of datasets and switch between modes (Walmsley and Kersten 2020). Aerial pixel-wise scene perception of the surrounding environment was designed with a Panoramic Annular Lens, a 360-degree annular FoV (Sun and Wang 2021). The team acquired a perspective panoramic scene dataset with annotated labels of track and better segmentation performance.

\subsection{Relevant Scholarship}

GoPro's is a leading company in exercise and outdoor shooting; it provided a platform for 360 panoramic photography. Many specific panoramic heads are designed for GoPro Hero 5, Hero 6, and Hero 7, such as the 
Panthers H5B. People can take high-resolution spherical 360 photos (Gopro-hero, 2017, 2019). Other post-processing tools including GoPro VR Reframe which straightens the horizon of 360 videos; and Insta360 Studio work with videos up to 5.7K (Figure 1) resolution using mp4 format. The app provides users with a more powerful way to manage their media and turns smartphones into remote controllers. The workflow requires 6 Micro SD cards to capture and waterproof, and shockproof out of the array. The shockproof devices keep aerial cameras stable through isolate impulse or vibration during aerial operations. The compacted shockproof device is made of rubber (Figure 2).

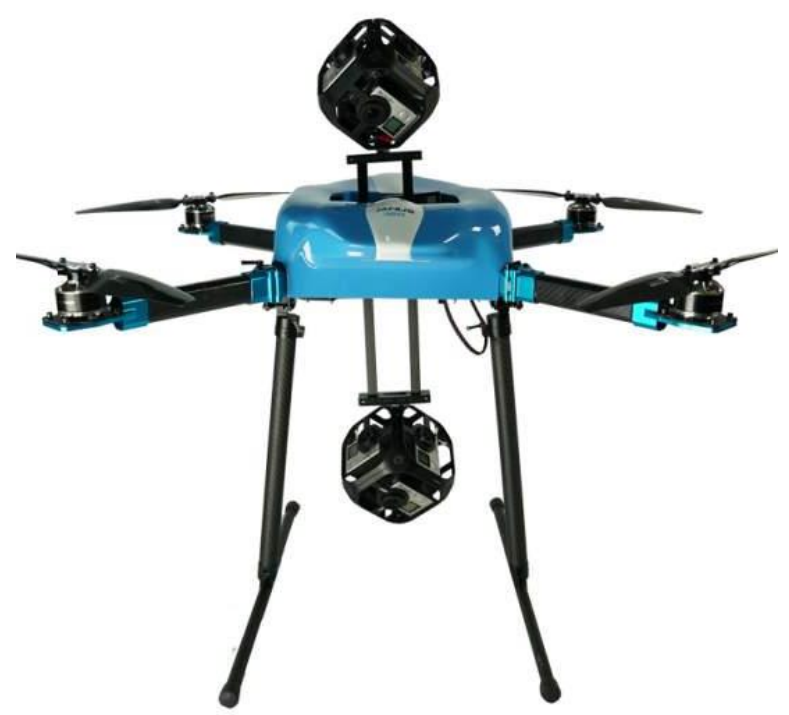

Figure 1. The foldable frame with a compact for 360 panoramic cameras set (https://veer.tv/blog/drone-with-camera-for-360-degree-aerial-photography/)

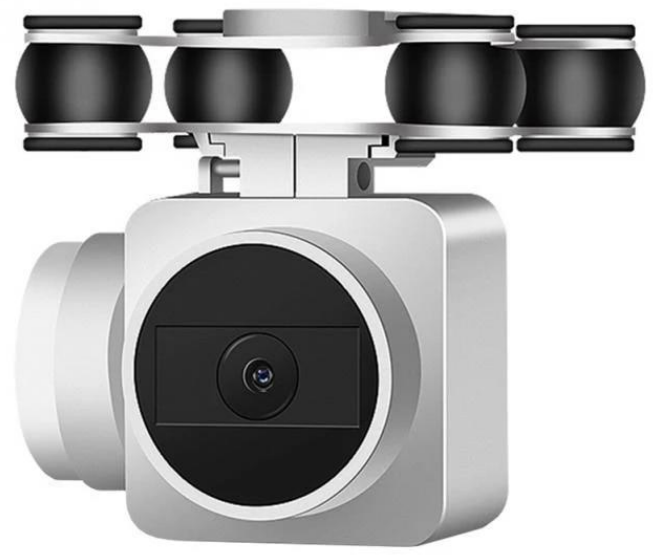

Figure 2. Shockproof aerial camera (Gopro, 2019)

Stabilizer aerial frame is also important for picture recording during moving (Merz 2017); wind and disturbances 
yields a more stabilized response. Some counter-balance structures could be a valuable way to stabilizing the position. The Ronin 2 use either mounted onto a drone with a motorized stabilization system using a payload of 30 pounds (Figure 3). High-torque motors allow the gimbal to resist winds and G-forces to maintains accuracy path (DJI, 2019).

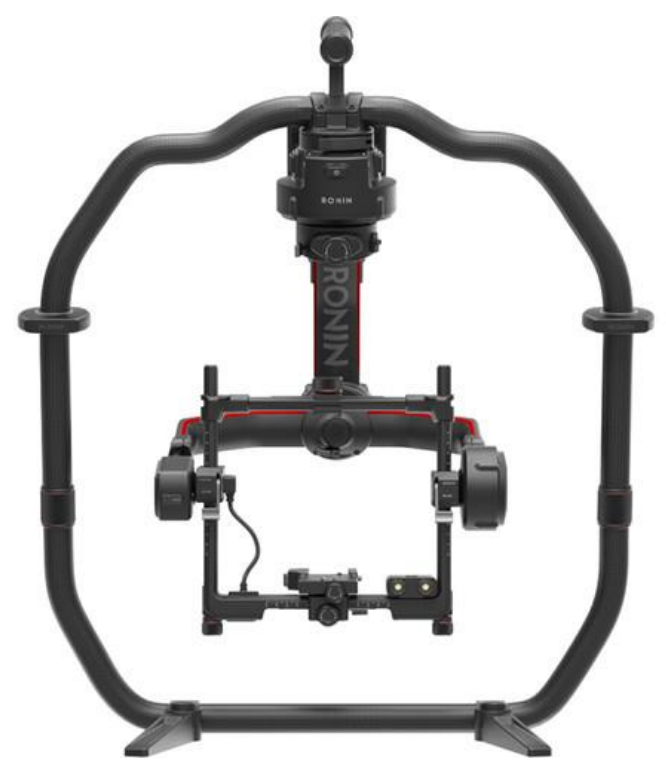

Figure 3. Stabilizer aerial camera frame (DJI, 2019)

\section{Design of Self-Assemble System}

\subsection{Explore Importance of the Problem}

VR equipment is constantly updated, and photographic equipment is also changing with each passing day, so designers need to find component information to suit system needs. The entry-level model of the camera only uses two fisheye lenses to shoot. The high cost of VR requires a large number of cameras (usually 6 to 12); after that, it takes much time to do post-processing to integrate relevant information into a data set that can be viewed. Photographic equipment also needs to consider the compatibility of post-production hardware and software.

The initial simple machine assembly is to place the aerial camera with the post on the camera, but it must be removed by post-processing if it shakes; this takes much time. The image quality of the simple machine is not good. GoPro has a complete set of cameras, equipment brackets, software, and the final Player, so the author chooses this system as the base.

We add the stabilizer to the machine table, and install objects of the same weight in the upper/ lower sections when assembling, with an adjustable pivot in the middle to support it (Figure 4). When the camera body shakes, the camera will still maintain a vertical position due to the balance. The shake of the aerial camera will not be transmitted to the camera.

The initially assembled system includes connecting rod hydraulic connecting rod to reduce camera shake. Today's three-axis stabilizer technology reduces instability through mechanical linkage; multiple rubber anti-vibration pads installed on the fuselage can reduce high-frequency amplification and reduce volume.

Since the photographic equipment installed on the drone protrudes below, additional brackets are needed to support it for takeoff. The bracket needs to be carried at any time, causing inconvenience for the photographer. We shortened the post of the camera to reduce the protrusion below. The video items captured by each camera need to be stitched and de-vibrated in the lower half of the image.

Since the aerial camera is above the camera, there will be a hole in the top when making the film; this situation will make the audience unhappy. Our processing method is to add a tiny camera on the top and combine the data of this camera with others to avoid the appearance of holes or foreign objects. The stitching of sky images is relatively easy to repair the image gap between the lenses. We usually join the two pictures with a gradient effect between the boundaries. 


\subsection{Solution Finding}

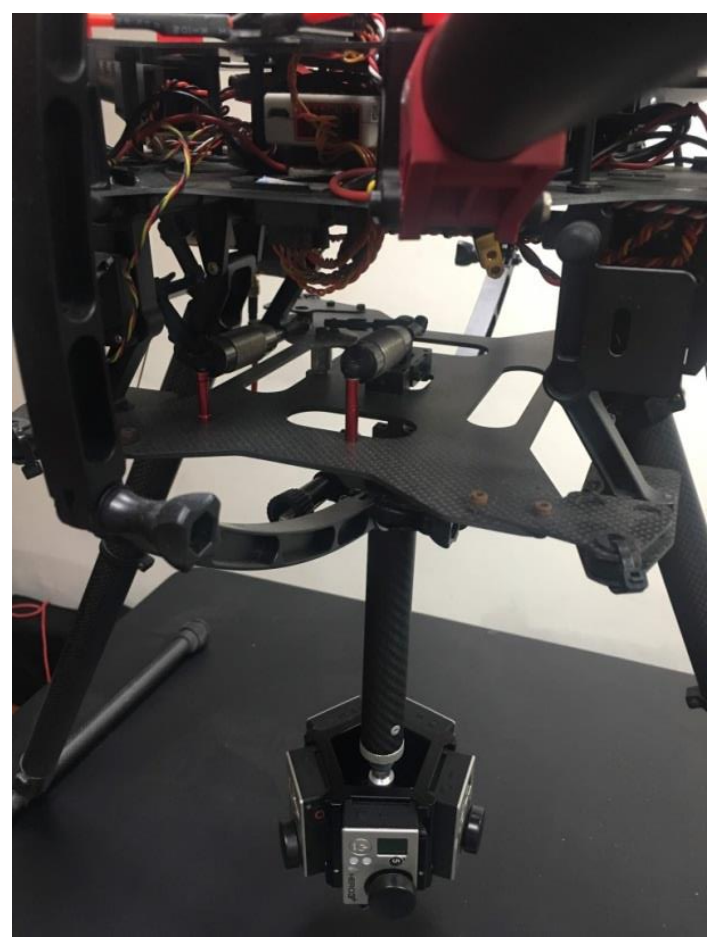

Figure 4. The detailed structure of the camera and stabilizer

We found that if the control is stable during these flights, user can hold it with hands when landing; this reduces the need for brackets (Figure 5). In addition, we often encounter strong winds when flying on the coast, which increases the load and power consumption. There have been two cases where the body crashed due to insufficient power storage, causing serious damage to the system. We think that this kind of experience is enough to inform the system of the residual power remotely, and the design of returning home will be helpful.

Nowadays, the miniaturized spherical structure holds cameras so that ordinary people can also control it. If the loading is reduced, and the total weight of the battery can also be reduced. Reducing the system load can undertake a more stable voyage mission. The well-known Insta Company has also developed a built-in CPU, which can pre-process the image in the body and transmit the image to the control terminal by wireless transmission. Use APP control to stitch the images, but only low-resolution images can be transmitted (preview mode).

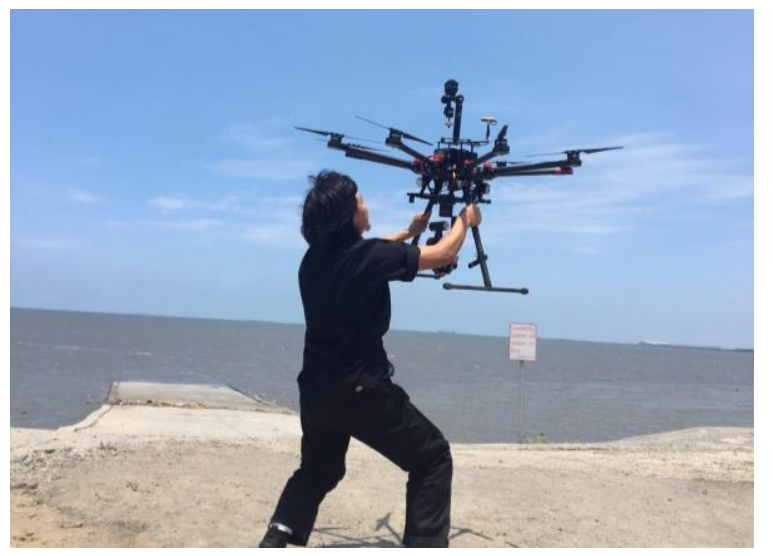

Figure 5. The takeoff and landing position of an aerial panoramic photographer 


\section{VR Film Construction}

\subsection{Image Post-Processor Available}

Image post-processing is about the combination and contrast of images to strengthen communication with the audience. We explain the connotation of the recorded images and the considerations of image post-processing as follows:

- Kolor-Autopano software applied for digital image processing: Post-production can considerably transform captured scenes, a remote $360^{\circ}$ employed to convey global landscape changing. GoPro HERO3+ motion recorders were installed to obtain $4 \mathrm{k}$ panoramic video.

- The video-capturing utilized an irrespective of lens rotation and tilting; the picture remains horizontal and faces the same direction. The $720^{\circ}$ VR display is a $360^{\circ}$ horizontal and vertical guide.

- Since the obviousness of visual symbols helps to attract people's attention.

Figure 6 shows a dual reversal image. We try to compare the effects of different image combinations, one of which is the reversal of the sky and the earth. As shown in Figure 6, we put the image of the industrial area on the bottom surface and then put the flipped farmland map on the top surface; the sky connects the two. We can feel the huge contrast between two different airspaces under the same sky from this way of displaying.

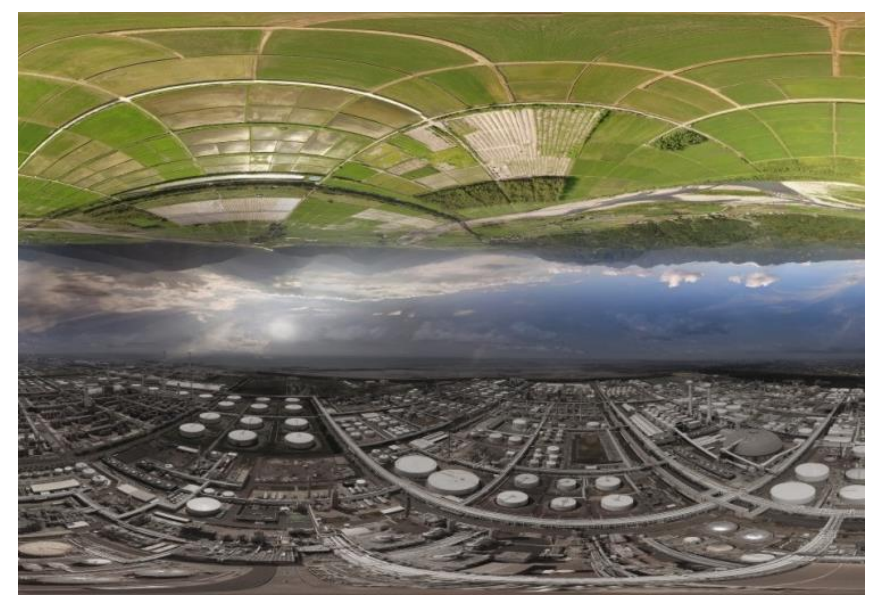

Figure 6. The upside-down view of the dual image (farmland/ industrial zone)

\subsection{Result of Image Process}

From aerial photography, space is condensed through the concept of planetary image transformation (Figure 7). We responded to the moral of the story of the little prince. People are trapped in the space of this lonely little planet, so we need to face up to acceptance and integration. 


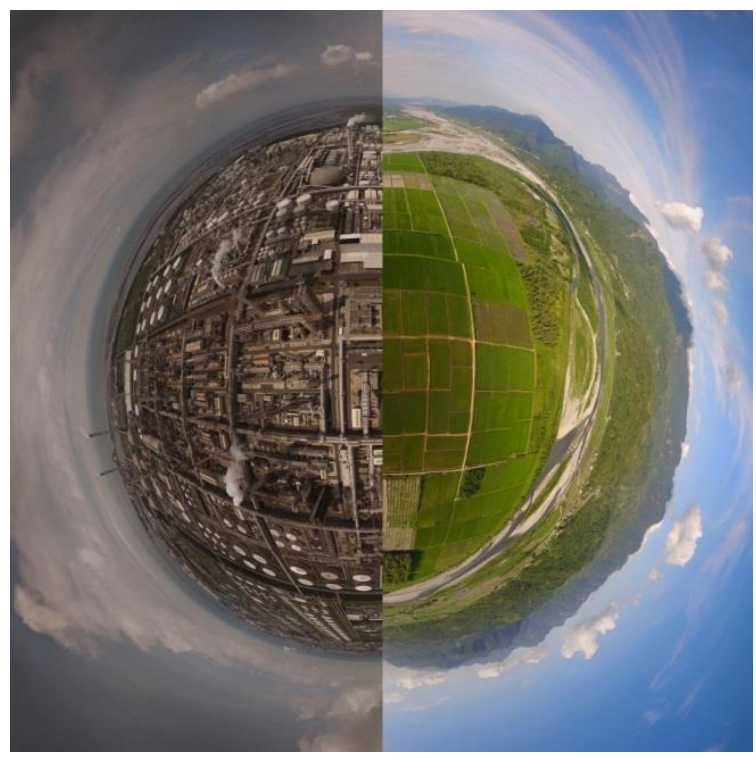

Figure 7. The sphere planet view of dual image

People think they are great, but in fact, they are trapped in the space we have created. We also apply it to the observation of people's lives to cause people to start thinking. For example, the different contrasts on the East Coast and the West Coast during the New Year, the combined hemispheres are used to highlight the reasons behind the differences.

We have seen the problem of stratum subsidence on the west coast. After the 1988 typhoon, the seawater inverted so that the entire cemetery and temple were immersed in the water. Through the conversion of aerial images, a sudden anomaly appeared. We have also captured images of houses and telephone poles immersed in water, and the audience expressed an indescribable pain. Reaching young people with images triggers their environmental awareness, which enhances the creator's sense of mission.

We also see that the landscapes of Kaohsiung Linyuan Industrial Zone, and Mailiao Industrial Zone, and the East Coast are pretty different. The strong contrast between the two sides shocked and realized that the huge industrialization process was beyond control. By being surrounded by coal cinders from coal-fired power plants, the immersive experience of aerial photography aroused inner shock. The residents of the seaside community have also shouted through the suffering of life over the past ten years.

VR videos need to be viewed by a viewer to be able to immerse them, and it is also convenient to explore so that problems can be presented from the discovery of images. When users cannot explore fascinating topics, they will be tired, so we have added sound to guide exploration.

\section{Large Scale Multi-Projector Displays}

The Taichung Fine Art Museum in Taichung City (Taiwan) provides financial support for displaying the environmental space. Because some people feel dizzy when wearing a helmet, some people are not happy to wear the same thing. We used multiple projectors to show this time in an ample space. The VR images were projected in the surrounding field, and the audience directly entered the field to experience it. This multi-projector technology has many new evolutions in recent years, the purpose of which is to be smooth at other connections. Radiometric and local light modulation needs reconsideration to integrate multiple projectors in a museum (Kim and Park 2020). The proposed system can enrich the museum exhibition by creating a virtual cultural object with a substantial 3D effect and high-fidelity appearance. The existing science museum's experience extended with a multi-faceted media facade through projection mapping and spectacular screen composition (Lee 2019).

Figure 8 shows two types of comparison: the sea area and the coastline/ground space. We put the sphere above in the center of the screen, and the three airspaces are connected to echo their corresponding spaces. We can see a group of high-hanging ring-shaped projection devices in the black part of the center of the picture, which is projected in different directions. However, the light source needs to be removed in the space to clear the image to be displayed. We can see in Figure 9 that the audience is quietly sitting or standing in the exhibition space, 
listening carefully to the explanation and experiencing the particular images brought by the aerial camera. In the follow-up interview after the visit, we evaluate to understand their feelings. All stakeholders can integrate and arouse attention to environmental protection issues.

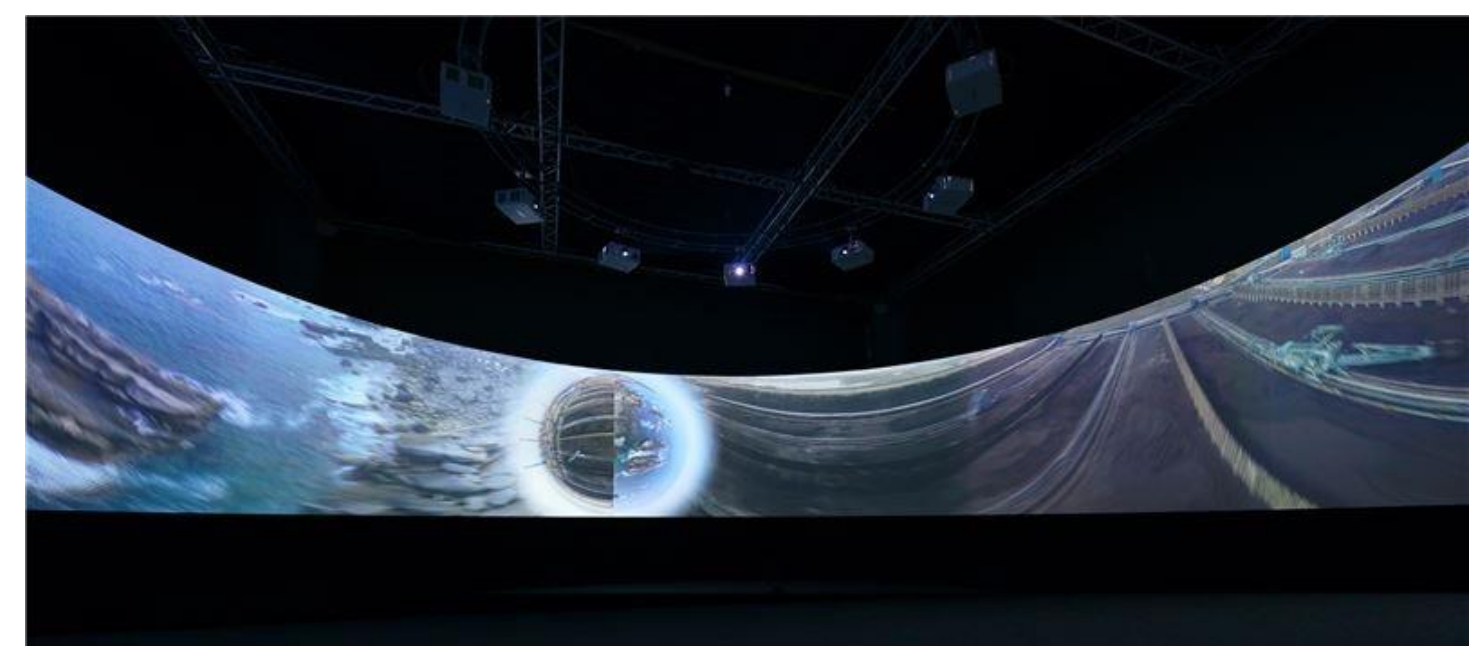

(a)

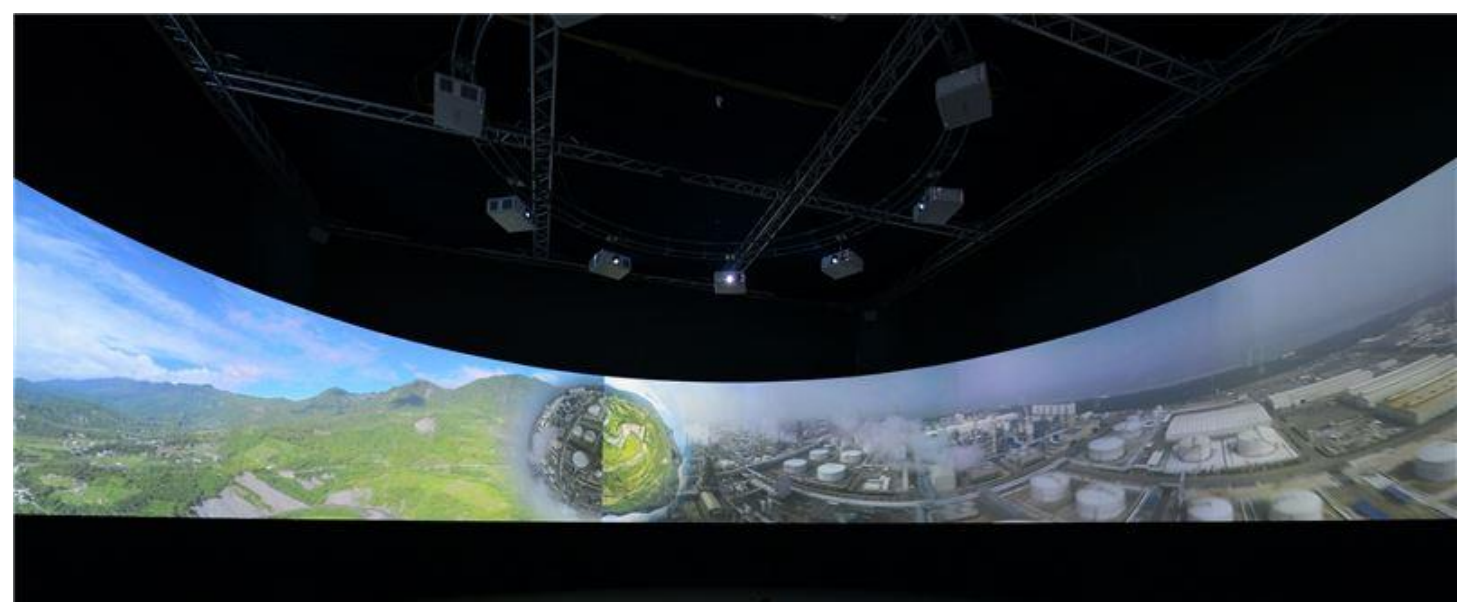

(b)

Figure 8. The multi-projector display installed in Taichung Fine Art Museum (a) sphere and contrast sea zone, (b) contrast farm zone 


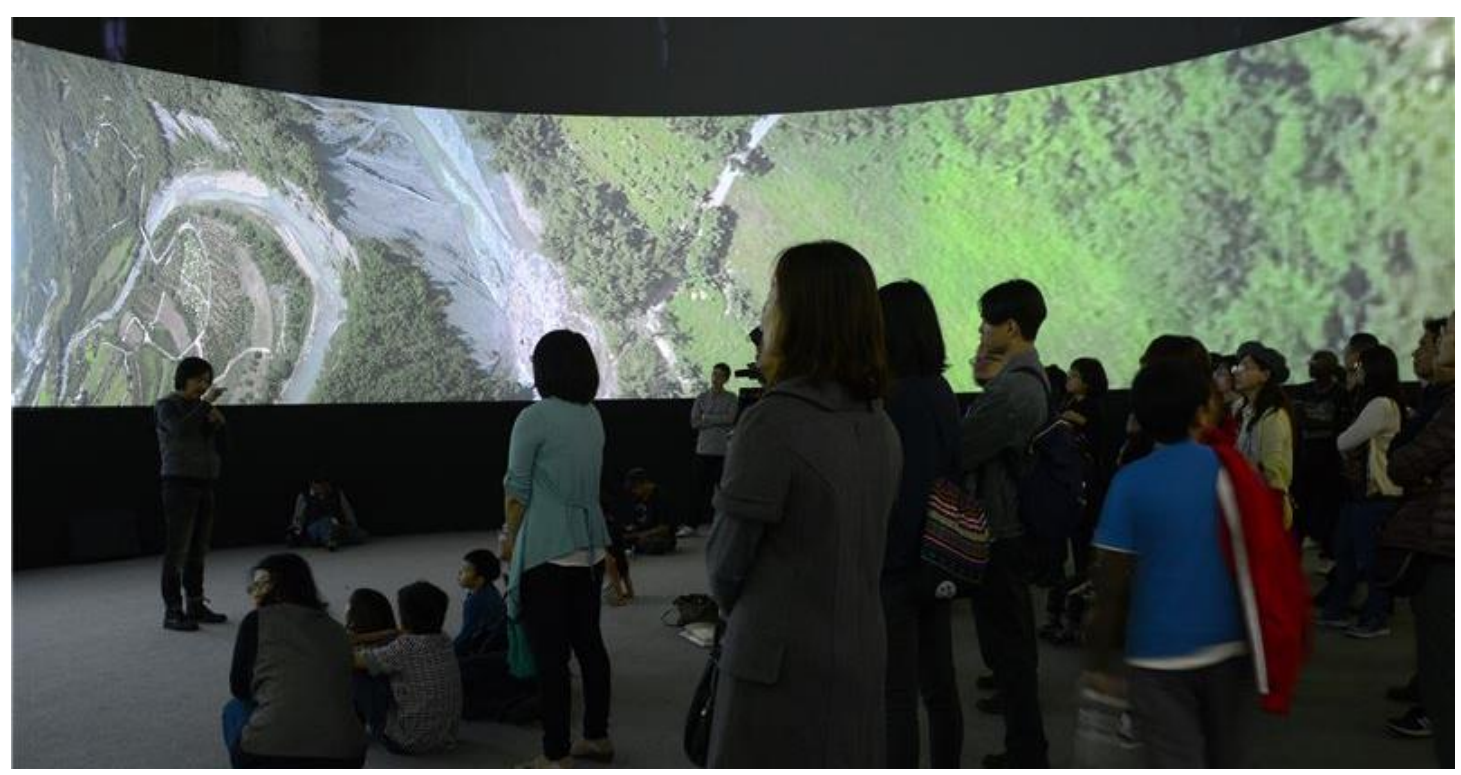

Figure 9. The people and guider experience VR image in Taichung Fine Art Museum

\section{Conclusions}

The researcher successfully designs a self-assemble system for VR aerial video. We shortened the post of the camera to reduce the protrusion below. The video items captured by each camera need to be stitched and de-vibrated in the lower half of the image. A tiny camera on the top of the drone combines the data of this camera with others to avoid the appearance of holes or foreign objects. Kolor-Autopano software applied for digital image processing: Post-production can considerably transform captured scenes, a remote $360^{\circ} \mathrm{employed}$ to convey global landscape changing. We used mathematical functions to project existing images into minor planets, condensing the three-dimensional space into a sphere, and successfully attracted attention during the display. The different contrasts on the East Coast and the West Coast, with the combined hemispheres, highlight the reasons behind the differences. Through the conversion of aerial images, a sudden anomaly appeared. We have also captured images of houses and telephone poles immersed in water, and the audience expressed an indescribable pain with the environmental scene. Images trigger young people's environmental awareness, which enhances the creator's sense of mission.

\section{Acknowledgements}

Feng thanks Taichung Art Museum and students for technology support and image processing efforts.

\section{References}

Alqahtani, H., \& Manolya Kavakli. (2017). IMAP-CampUS: Developing an intelligent mobile augmented reality program on campus as a ubiquitous system. In Proceedings of the 9th International Conference on Computer and Automation Engineering, 1-5.

Andri, C., Mohammed Hazim Alkawaz, \& Safa Riyadh Waheed. (2019). Examining effectiveness and user experiences in 3d mobile-based augmented reality for msu virtual tour. In 2019 IEEE International Conference on Automatic Control and Intelligent Systems (I2CACIS), 161-167. IEEE.

DJI.

(2019).

Retrieved

from

https://www.bhphotovideo.com/c/product/1333927-REG/dji_ronin_2_3_axis_stabilizer.html

Feng, W. J., Yao-Chi Mu, \& Fang-Lin Chao. (2019). 360 ${ }^{\circ}$ Panoramic Aerial Creation and Environmental Awareness. In 2019 IEEE 2nd International Conference on Knowledge Innovation and Invention (ICKII), 217-220. IEEE.

Gopro-hero. (2017). Retrieved from https://360rumors.com/gopro-hero-360/

Gopro-hero. (2019). Retrieved from https://gopro.com/

Kim, T. W., \& Nam-Ki Park. (2020). Research and development of interactive exhibition contents for sound light exhibition space in Science Museum. Journal of the Korea Convergence Society, 11(7), 137-144. 
Lee, Y. Y., Jong Hun Lee, Bilal Ahmed, Moon Gu Son, \& Kwan H. Lee. (2019). A new projection-based exhibition system for a museum. Journal on Computing and Cultural Heritage (JOCCH), 12(2), 1-17.

Merz, M. (2017). A strategy for robust precision control of an endbody being towed by an orbiting UAV. In AIAA Guidance, Navigation, and Control Conference, 1040.

Sun, L., Jia Wang, Kailun Yang, Kaikai Wu, Xiangdong Zhou, Kaiwei Wang, \& Jian Bai. (2021). Aerial-PASS: panoramic annular scene segmentation in drone videos. arXiv preprint arXiv:2105.07209, 2021.

Walmsley, A. P., \& Thomas P. Kersten. (2020). The Imperial Cathedral in Königslutter (Germany) as an immersive experience in virtual reality with integrated $360^{\circ}$ panoramic photography. Applied Sciences, 10(4), 1517-1523.

Zhu, Y., Jiao Baocong, \& Pang Xin. (2018). The research on campus panoramic system based on VR technology. Science Journal of Education, 6(2).

\section{Copyrights}

Copyright for this article is retained by the author(s), with first publication rights granted to the journal.

This is an open-access article distributed under the terms and conditions of the Creative Commons Attribution license (http://creativecommons.org/licenses/by/4.0/). 